\title{
Different profiles of body mass index evolutions among patients with multidrug-resistant tuberculosis: a retrospective cohort study.
}

\section{Alhassane Diallo}

INSERM, U1137, CIC-EC 1425, Department of Biostatistics, Clinical investigation, Phamaceutics in Infectious Disases, University Paris Diderot, Paris

Boubacar Djelo Diallo ( $\sim$ diallodjelo@yahoo.fr)

Universite Gamal Abdel Nasser de Conakry https://orcid.org/0000-0002-3015-6722

\section{Lansana Mady Camara}

Faculté des sciences et techniques de la santé, université gamal abdel nasser de Conakry, service de pneumo-phtisiologie, CHU Conakry, Hôpital National Ignace Deen

\section{Lucrèce Ahouéfa Nadège Kounoudji}

Faculté des sciences techniques de la santé, Université gamal abdel nasser de Conakry

\section{Boubacar Bah}

Centre antituberculeux de la Carrière, Conakry

\section{Fulgence N'Zabintawali}

Laboratoire National des mycobactéries, Conakry

\section{Miguel Carlos-Bolumbu}

Urgences réanimation centre hospitalier Sud Essonnes CHSE, Paris

\section{Mamadou Hassimiou Diallo}

Centre population et développement, Institut de recherche pour le développement, Université Paris Descartes, Paris

\section{Oumou Younoussa Sow}

Faculté des sciences et techniques de la santé, Université gamal abdel nasser de Conakry

\section{Research article}

Keywords: BMI, Multidrug-resistant, Tuberculosis, subgroup

Posted Date: November 7th, 2019

DOI: https://doi.org/10.21203/rs.2.16913/v1

License: (c) (i) This work is licensed under a Creative Commons Attribution 4.0 International License. 
Version of Record: A version of this preprint was published at BMC Infectious Diseases on April 28th, 2020. See the published version at https://doi.org/10.1186/s12879-020-05028-0. 


\section{Abstract}

Background Despite the predictor role of the body weight variation on multidrug-resistant tuberculosis (MDR-TB) treatment outcome, little data are available to corroborate this finding. We aimed to study the course of weight in patients with MDR-TB, to identify subgroups of weight evolutions, and to determine factors that influence these evolutions.

Methods Patients treated with a shorter MDR-TB treatment regimen between June 07, 2016 and June 22, 2018 from three major drug-resistance TB centres in Guinea, who had rifampicin resistance, and who were cured or died were analysed. Patients were seen monthly until the end of treatment. Clinical outcome was the Body Mass Index (BMI). We used a linear mixed model to analyze the course of BMI and a latent class mixed model to identify subgroup of BMl evolutions.

Results Of 232 patients treated for MDR-TB during the study period, 165 (71\%) were analysed. These patients had a total of 1387 visits, with a median of 5 visits (interquartile range, $3-8$ visits). Monthly $\mathrm{BMI}$ increase was 0.24 (SE 0.02) per $\mathrm{kg} / \mathrm{m} 2$. Factors that associated with faster BMI progression were cured to MDR-TB treatment (0.24 [SE 0.09] per $\mathrm{kg} / \mathrm{m} 2 ; \mathrm{p}=0.0205)$, and the absence of lung cavities on X-ray (0.18 [0.06] per $\mathrm{kg} / \mathrm{m} 2 ; \mathrm{p}=0.0068)$. Two subgroups of BMI evolution were identified: "Rapid BMI ( $=121 ; 85 \%)$ and "Slow BMI evolution $(n=22 ; 15 \%)$. Patients in the slow increasing BMI group were mostly female (68\%) without history of TB treatment (41\%) with most severe clinical condition at baseline, characterized by a higher frequency of symptoms including HIV infection (59\%), depression (18\%), dyspnea (68\%), poor adherence to MDR-TB treatment (64\%), lower platelets count, and higher liver SGOT count. These patients had also a longer time to-initial culture conversion delay (log-rank test: $p=$ 0.0087).

Conclusion The available data provide quantitative information on BMI progression of patients with MDR-TB treated with a shorter regimen, and allowed the identification of the subgroup of patients with different BMI evolutions. Furthermore, they emphasize the usefulness of BMI as biomarker to monitor MDR-TB treatment outcome.

\section{Introduction}

Multidrug-resistant tuberculosis (MDR-TB) is defined as resistance at least one of the two powerful antiTB drugs, that are isoniazid (INH) and rifampicin (R). In 2016, the World Health Organization (WHO) reported 600000 new cases of MDR-TB globally [1] with 136 cases from Guinea (Conakry) [2].

In a context of limited resources like Guinea, the identification of a biomarker of the MDR-TB treatment response that is easily measured and accessible in clinical practice would be beneficial both for the management of patients and the tuberculosis control programs. Numerous studies have been shown the impact of malnutrition measured by the weight on the poor MDR-TB treatment outcome, and suggested that weight loss is a potential biomarker of treatment response [3-7]. Moreover, a longitudinal study showed that a variation of the weight during the first 6 months differed according to the treatment 
outcome, with decreasing weight over time in patient with poor treatment outcome [7]. The underlying hypothesis of the mixed linear model used to analyze weight change in this study, is that all patients have similar variations in weight over time. Because, patients with MDR-TB often differed in the severity of the disease at baseline as clinical presentation, the number of episodes of TB, the presence of HIV coinfection or comorbidity (diabetes), the susceptibility to drugs toxicity and resistance, this assumption of homogeneity of weight change seem too strong. Hence the need to identify homogeneous subgroups of patients with different weight evolutions and to characterize them, which would better target at-risk patients to ensure more efficient use of resources and optimal patient management.

To our best knowledge, no study has reported the subgroups identification of weight change in patients with MDR-TB. Here, we reported data from a retrospective cohort study of patient treated with a standardized shorted treatment regimen of 9-months in Guinea. We aimed (a) to specify the course of weight gain as well as factors that influence the weight course, (b) identify different subgroups of weight evolution, and (c) determine the factors associated with weight subgroups evolutions.

\section{Methods}

\section{Study design and population}

A retrospective multicenter longitudinal cohort study from three referral drug-resistance TB centres in Guinea (Centre Hospitalier Universitaire Ignace-Deen, Carrière and Tombolia) was conducted. Patients with rifampicin resistance who were died from any reason or who were declared as cured according to the 2013 WHO recommendation [8] to a shorter MDR-TB treatment regimen between June 07, 2016 and June 22, 2018, were analysed (figure 1). Drug-resistance diagnosis was done by the Xpert MTB/RIF test. According to the guideline for the MDR-TB management in Guinea ${ }^{2}$, naive patients for second-line anti-TB drugs were treated with a short 9-month regimen, consisting of an intensive phase lasting a minimum of 4 months including moxifloxacin, kanamycin, clofazimine, prothionamide, pyrazinamide, ethambutol, and $\mathrm{INH}$ at high dose. The intensive phase was then followed by the continuation phase during 5 months and consisting of administration of four drugs: moxifloxacin, clofazimine, pyrazinamide, and ethambutol. Patients were seen at baseline and followed by monthly visit for 9 months. The study was approved by the National Ethics Committee for Heath Research (NECHR) attached to the Ministry of Health (Conakry, Guinea).

\section{Outcome and predictors variables}

Clinical outcome was the body mass index (BMI) calculated with the formula [weight/(height) ${ }^{2}$ ]. Weight, sputum smear and sputum culture were obtained monthly during the MDR-TB treatment course. Sputum smear conversion was defined as two consecutive negative sputum smears taken at least 30 days apart following an initial positive sputum smear. Similarly, culture conversion was defined as two consecutive negative culture taken at least 30 days apart following an initial positive sputum culture. Time-to initial 
sputum smear (or culture) conversion was defined as the time in months from the date of start of MDRTB treatment to the date of specimen collection for the first of two consecutive negative sputum smears (or culture) results, even if a subsequent positive sputum smears (or culture) result occurred later. Demographic and clinical data included age, gender, residence, HIV infection status, history of the previously treated TB, the presence of cavities on chest X-ray determined by the senior radiologist, baseline data on weight, clinical symptoms (as chest pain or cough), depression status, treatment adherence, and biological data. Information was collected using a case report form (CRF) from the MDRTB registry.

\section{Statistical analysis}

Descriptive statistics (frequencies and percentage or mean and standard deviation [SD]) were used to describe demographic and clinical characteristics of participants at baseline. BMI at baseline was compared between treatment outcome (cured vs died) using the Student t-test. To analyze the change of BMI over time, we applied the linear mixed (LM) model [9] with correlated auto-regressive intercept and slope random-effects to account for inter-patients variability. Time from MDR-TB initiation in months was used as the time scale. Both linear, quadratic and cubic effects of time were tested, and then the model that best fitted the data was choose via likelihood test ratio. To identify factors that affected the BMI progression, we tested the interaction between time variable and the given factors. Predictors with a pvalue less than 0.10 in the univariate analysis, including the interactions terms were entered into a multivariate regression. Independent predictors that influenced the BMI progression rate were selected through a backward procedure based on the lowest Akaike information criterion.

To identify subgroups of participants exhibiting different trajectories of BMI, a latent class mixed (LCM) model that account for individual and latent group structure variability through random-effects was used [10]. The same fixed and random-effects included in the LM model were used for the LCM model. Independent predictors that affected the BMI evolution from LM model were used to explain groups membership. The best-fitting model with the optimal number of latent classes was selected using the compromise between the lower integrated classification likelihood with Bayesian information criterion (ICL-BIC) and the mean posterior probabilities belonging to each latent class above $0.70[10,11]$. For each patient, the probability of belonging to each different trajectory was determined: each patient was then assigned to the group for which her/his probability belonging to a trajectory was the highest.

Distributions of the baseline factors across these classes were compared a posteriori using a chi-squared test for the categorical variables and Student t-test for the continuous variables. To assess whether the time-to initial sputum smear and culture conversions was directly related to these classes, the KaplanMeier method was used and differences in survival time were compared with a log-rank test. All data analyses were done in $\mathrm{R}$ (version 3.5.1). Significance was defined as a $\mathrm{p}$-value less than 0.05 , and all tests were two-sided.

\section{Results}




\section{Clinical characteristics at baseline}

Of 232 patients with MDR-TB, a total of 165 patients who meet the inclusion criterion were analyzed. These patients had a total of 1387 visits, with a median of 5 visits (interquartile range, $3-8$ visits). The patients mean age was 34.0 (SD 11.3) years, with the majority of them were male (67\%), leaving in urban area (74\%), with a mean BMI of $17.5(2.7) \mathrm{kg} / \mathrm{m}^{2}$. Most patients presented cough (94\%), $42(26 \%)$ patients were HIV positive, and $13(9 \%)$ patients had cavities on initial chest X-ray. According to the history of TB, 134 (81\%) patients were previously treated, $88 \%$ and $83 \%$ had a positive baseline sputum smear and culture respectively. At the study end, $92 \%$ (108/118) and $89 \%(105 / 118)$ of patients converted their sputum smear and culture in a median of 59 days (interquartile range: 31-61 days) respectively. Overall, $81 \%$ (133/165) of patients were cured to the MDR-TB treatment, and 19\% (32/165) died for any reason. Table 1 detailed the study population according to the treatment outcome.

\section{Rates of BMI change}

BMI increase data was best fitted with a linear model (figure 2 B). Overall, the monthly increase BMI was 0.24 (SE 0.02) per $\mathrm{kg} / \mathrm{m}^{2}$. Table 1 from appendix showed factors associated to increase BMI over time from univariate analysis. To identify baseline factors that were independently associated with faster increase of BMl, we applied a multivariable modelling. Cure to the MDR-TB treatment (0.24 [SE 0.09] per $\left.\mathrm{kg} / \mathrm{m}^{2} ; \mathrm{p}=0.0205\right)$, and the absence of lung cavities on X-ray $\left(0.18[0.06]\right.$ per $\left.\mathrm{kg} / \mathrm{m}^{2} ; \mathrm{p}=0.0068\right)$ were associated with faster increase in BMI. The corresponding predictions and their $95 \%$ confidence interval (Cl) were displayed in figure 2 ( $C$ and $D)$. While the increase $B M I$ was faster in patients who declared as cured, the course of BMI decreased in whom died (figure $2 \mathrm{C}$ ).

\section{Trajectories of BMI and their relationship to baseline factors and sputum (smear and culture) conversion}

To identify different trajectories of BMI, the LCM model with several latent classes, ranging from 1 to 4 , were estimated (appendix). The membership of these classes was explained by the treatment outcome and the lung cavities on X-ray. The model with the optimal number of classes selected by the compromise criterion included two different BMI evolutions (figure 3 ). Class $1(n=121,85 \%)$ was characterized by faster BMI evolution over time, named as "Rapid BMI evolution"; and Class 2 ( $n=22$, $15 \%$ ) correspond to patients who had slow BMI evolution over time, named as "Slow BMI evolution". According to the posterior classification provided in table 3, the patients in the "Slow BMl evolution" group were more severely affected at baseline. These patients were mostly female (68\%) without history of TB treatment $(41 \%)$ who had a higher frequency of symptoms including positive HIV infection (59\%), depression (18\%), dyspnea (68\%), nausea (23\%), poor adherence to MDR-TB treatment (64\%), lower platelets count (377.7 [181.2]), and higher liver SGOT count (32.7 [9.5]). These patients had also a longer time to-initial culture conversion delay (figure $4 \mathrm{~B}$, log-rank test: $p=0.0087$ ), while their time to-initial 
sputum smear conversion was comparable to those patients in rapid BMI evolution group (figure 4A, $p=$ 0.6562).

\section{Discussion}

To our best knowledge, this is the first study to address the identification of subgroups of weight evolutions and to determine factors associated with these subgroups.

Two profiles of BMI evolutions were identified: rapid and slow, with the average probability of belonging to the two LCM model being higher, ranging from 0.82 to 0.99 , suggesting unambiguous classification (appendix). From a linear mixed (LM) model, we found that a BMI increase over time, with different evolution according to the treatment outcome. After controlling for lung cavities on X-ray, patients who were cured had gained on average $2.62 \mathrm{~kg} / \mathrm{m}^{2}$ of BMI at the treatment end. A similar result was previously reported that patients who were cured had gained on average $3.9 \mathrm{~kg}$ at the end of the sixth month [7]. Unlike the LM model, which shows an average gain BMI over time, our subgroup analysis showed that the speed of this weight gain was not identical for all patients. The most interesting finding was that the patients in the slow BMI evolution had poor response to the MDR-TB treatment, suggesting that weight may serve as a potential biomarker to monitor treatment outcome. These patients were characterized by a positive HIV infection, depression symptom, poor adherence to the MDR-TB treatment, and delay to the culture conversion. This is a relevant finding in public health, particularly in resourcelimited settings because they allow a better targeting patient with high-risk to the treatment failure, and to focus the resources needed to improve treatment success rates. Strategies as close monitoring of these patients with therapeutic education to improve treatment adherence, the setting up of psychiatric consultations to manage depression will be contribute to improve the prognosis of these patients and increase their chance of success.

Furthermore, in patients with slow BMI progression, the chance of culture conversion was reduced by $65 \%$ $(\mathrm{HR}=0.35,95 \% \mathrm{Cl}[0.13-0.96] ; \mathrm{p}=0.0087)$. This finding was higher than those reported from studies evaluating the impact of baseline weight loss $\left(\mathrm{BMl}<18.5 \mathrm{~kg} / \mathrm{m}^{2}\right)$ and delay in culture conversion $[4,5]$. The reduce chances of culture conversion were $43 \%$ and $45 \%$ for Indonesian and South Korean patients respectively. In addition, $89 \%$ of our patients converted their culture in a median of 2 months, which was higher compared to the rates of culture conversion reported in Indonesia $(80 \%)^{5}$ and South Korea (70\%) [4], suggesting the reasonably well performance of our MDR-TB treatment program.

Recently, the superiority of the culture conversion than smear conversion in predicting MDR-TB treatment outcomes was demonstrated with an optimum times points between four and six months after treatment commencement. This conclusion supports the $\mathrm{WHO}$ recommendation to add culture examination to the sputum smear for the monitoring of MDR-TB patients for a better prediction of successful treatment outcomes [12]. Nevertheless, in resources-constrained settings, the sputum culture is resource intensive, takes time to obtain, costly, requires specialized laboratories, equipment and trained staff. We found that in patient with MDR-TB, the stable or decreases weight between two visits is probably a sign of a poor 
response to treatment, especially in an HIV-infected, depressed woman with lung cavities on X-ray, and who had a poor treatment adherence. Since the measure of body weight is an easy, rapid, inexpensive method, and accessible everywhere, the association between a faster increase in BMI and shorter time-to initial culture conversion suggest the useful of weight assessment as a surrogate of the culture conversion to predict an early MDR-TB treatment response.

The mechanism underlying weight loss in patients with MDR-TB is well known [13]. Poverty-induced malnutrition is one of the main causes of weight loss in countries with high prevalence of TB such as Guinea. By decreasing the concentration of immunoglobulins, interleukin-2 receptor, and T-cell subset (helper, suppressor-cytotoxic, and natural killer cells) [14], malnutrition further alters the immunity of patients with TB, making them vulnerable to infections such as HIV, and prone to severe clinical presentation and a higher proportion of positive sputum cultures. Effective poverty alleviation strategies such as reducing social inequalities, access to safe drinking water, and improving the nutritional status of patients will reduce the post-treatment community transmission and result in better success rates.

The current study has a number of strengths. First, patients were evaluated from a three-referral center for MDR-TB management in Guinea, which reduce selection bias and increase the validity of the extrapolation of our findings to the entire population of Guinean patients with MDR-TB. Second, unlike to the conventional mixed linear model used to describe the evolution of weight over time, our subgroup analysis identified a group of patients with poor prognosis (slow BMI evolution) as well as the characteristics of these patients. Third, we used a compromise criterion to select the best BMI subgroups evolutions instead of using only the Bayesian information criterion. As mentioned above, model with two classes has a higher average posterior probability up 0.80 , suggesting unambiguous classification. Fourth, to account of informative dropout, we applied a sensitivity analysis using a joint model for longitudinal and time-to dropout [15]. The results obtained from this joint model were similar to estimations using standard linear mixed model, suggesting an absence of bias in parameter estimations (data not shown). However, retrospective design, small sample size, some missing factors such as diabetes status, smoking and alcohol use, information on second-line drugs susceptibility, and other anthropometric measurements, such as skin-fold thickness or waist circumference, which could possibly serve proxy to weight assessment were limits of this study. Nevertheless, further prospective cohort study with large sample could be needed to confirm these findings.

\section{Conclusion}

The current data allowed us to identify two different groups of BMI evolution and provided evidence of an association between weight progression and treatment outcome. These findings suggest monitoring of body weight as a potentially useful surrogate of sputum culture conversion in predicting successful MDRTB treatment outcome, because patients who had rapid BMI progression were more like to culture conversion. Furthermore, these findings suggest also that the management of HIV infection and depression status, as well as more therapeutic education to improve treatment adherence may be reduce the risk for community transmission from patients with MDR-TB. In addition, the results provide more 
information to help with patient's selection and stratification for the design of future interventional clinical trials.

\section{Abbreviations}

BMI: body mass index; Cl: confidence interval; CRF: case report form; HIV: human immunodeficiency virus; INH: isoniazid; ICL-BIC: integrated classification likelihood with Bayesian information criterion; LMM: linear mixed model; LCMM: Latent class mixed model; MDR-TB: Multidrug-resistant tuberculosis; PV: predictive value; HR: hazard ratio; SD: standard deviation; SE: standard error; TB: tuberculosis; WHO: World Health Organization.

\section{Declarations}

\section{Ethics approval and consent to participate}

The study was approved by the National Ethics Committee for Heath Research (NECHR) attached to the Ministry of Health (Conakry, Guinea). The study was based in accordance to the Declaration of Helsinki, and the confidentiality of the data was guaranteed.

\section{Consent for publication}

Not applicable

\section{Availability of data and materials}

The data is available upon authors request.

Competing interests

We declare no competing interests in relation with this work.

\section{Funding}

None

\section{Author's contributions}

AD conceived the study design, analysed the data, and drafted the manuscript, BDD contributed to the conception, organization the research project, supervision data collection, and critical revision to manuscript, KLAN collected data, LMC, OYS, contributed to the conception, organization the research project, and commented the manuscript, BB, FNZ, MCB, and MHD commented the manuscript. All authors approved the final version of the manuscript. 
We thank the National Tuberculosis Control Program for their collaboration, and Action Damien Foundation for their patients support during treatment.

\section{References}

1. World Health Organization: Global tuberculosis report 2017. Geneva, Swizerland: WHO press, 2017.

2. National Tuberculosis Control Program Guinea: Annual report of TB control activity, 2018.

3. Podewils LJ, Holtz T, Riekstina V, Skripconoka V, Zarovska E, Kirvelaite G, et al. Impact of malnutrition on clinical presentation, clinical course, and mortality in MDR-TB patients. Epidemiol Infect. 2011; 139:113-20.

4. Park H-O, Kim S-H, Moon S-H, Byun J-H, Kim J-W, Lee C-E, et al. Association between Body Mass Index and Sputum Culture Conversion among South Korean Patients with Multidrug Resistant Tuberculosis in a Tuberculosis Referral Hospital. Infect Chemother. 2016; 48:317.

5. Putri FA, Burhan E, Nawas A, Soepandi PZ, Sutoyo DK, Agustin H, et al. Body mass index predictive of sputum culture conversion among MDR-TB patients in Indonesia. Int J Tuberc Lung Dis. 2014; 18:564-70.

6. Cegielski P, Gler MT, Guilatco R, Johnson JL, Caoili JC, Ershova J. Weight Gain and Response to Treatment for Multidrug-Resistant Tuberculosis. Am J Trop Med Hyg. 2013; 89:943-9.

7. Chung-Delgado K, Revilla-Montag A, Guillén-Bravo S, Bernabe-Ortiz A. Weight variation over time and its relevance among multidrug-resistant tuberculosis patients. Int J Infect Dis. 2014; 23:20-4.

8. WHO: Definitions and reporting framework for tuberculosis-2013 revision (updated 2014). Geneva: Switzerland World Health Organization; 2013.

9. Verbeke G, Molenberghs G. Linear Mixed Models for Longitudinal Data. Dordrecht: Springer; 2009

10. Commenges D, Jacqmin-Gadda H. Dynamical biostatistical models. Boca Raton: CRC Press/Taylor \& Francis; 2016. 374 p.

11. Han J, Slate EH, Peña EA. Parametric latent class joint model for a longitudinal biomarker and recurrent events. Stat Med. 2007; 26:5285-302.

12. Alene KA, Viney K, Yi H, McBryde ES, Yang K, Bai L, et al. Comparison of the validity of smear and culture conversion as a prognostic marker of treatment outcome in patients with multidrug-resistant tuberculosis. Hasnain SE, editor. PLOS ONE. 2018; 13:e0197880.

13. Byrd, Jr. RP, Mehta JB, Roy TM. Malnutrition and Pulmonary Tuberculosis. Clin Infect Dis. 2002; 35:634-5.

14. Scrimshaw NS, SanGiovanni JP. Synergism of nutrition, infection, and immunity: an overview. Am J Clin Nutr. 1997; 66:464S-477S.

15. Rizopoulos D. Joint models for longitudinal and time-to-event data: with applications in R. Boca Raton: CRC Press; 2012. 261 p.

\section{Tables}


Table 1: Population characteristics at baseline

\begin{tabular}{|c|c|c|c|}
\hline & \multicolumn{3}{|c|}{ MDR-TB treatment outcomes } \\
\hline & Total $(n=165)$ & Cured $(n=133)$ & $\operatorname{Dead}(n=32)$ \\
\hline Age at baseline & $33.3(11.6)$ & $32.2(10.5)$ & $37.5(14.7)$ \\
\hline Sex (male) & $111(67.3)$ & $97(72.9)$ & $14(43.8)$ \\
\hline Residence (Urban) & $122(73.9)$ & $37(27.8)$ & $6(18.8)$ \\
\hline BMl & $17.5(2.7)$ & $17.8(2.6)$ & $16.0(2.7)$ \\
\hline Initial sputum smear (positive) & $145(87.9)$ & $120(90.2)$ & $25(78.1)$ \\
\hline Initial sputum culture (positive) & $137(83.0)$ & $113(88.9)$ & $24(85.7)$ \\
\hline HIV status (positive) & $42(25.6)$ & $27(20.5)$ & $15(46.9)$ \\
\hline Lung Cavities on X-ray (yes) & $13(9.1)$ & $9(7.7)$ & $4(15.4)$ \\
\hline History of TB treatment (previously treated) & $134(81.2)$ & $114(85.7)$ & $20(62.5)$ \\
\hline Treatment adherence (yes) & $144(87.3)$ & $128(96.2)$ & $16(50.0)$ \\
\hline Depression (yes) & $8(4.9)$ & $2(1.50)$ & $6(18.8)$ \\
\hline Chest pain (yes) & $73(44.2)$ & $54(40.6)$ & $19(59.4)$ \\
\hline Cough (yes) & $156(94.6)$ & $127(95.5)$ & $29(90.6)$ \\
\hline Dyspnea (yes) & $52(31.5)$ & $34(25.6)$ & $18(56.3)$ \\
\hline Nausea (yes) & $13(7.9)$ & $7(5.3)$ & $6(18.8)$ \\
\hline Vomiting (yes) & $17(10.3)$ & $10(7.5)$ & $7(21.9)$ \\
\hline Hemoglobin count & $10.7(2.2)$ & $10.8(2.1)$ & $10.0(2.6)$ \\
\hline Platelets count & $388.0(159.9)$ & 396.1 (152.4) & $353.8(187.1)$ \\
\hline Lymphocytes count & $1.9(1.4)$ & $1.8(0.9)$ & $2.0(2.6)$ \\
\hline Neutrophiles count & $5.0(2.6)$ & $5.0(2.6)$ & $5.5(2.7)$ \\
\hline Creatinine count & $77.7(19.6)$ & $76.8(19.5)$ & $81.3(19.7)$ \\
\hline Live SGOT count & $29.6(5.4)$ & $29.2(3.8)$ & $31.2(9.3)$ \\
\hline Liver SGPT count & $32.2(5.0)$ & $32.2(4.2)$ & $32.3(7.6)$ \\
\hline
\end{tabular}

Data are expressed as $n=$ number (\%) for categorical variable, and mean (SD); SD = standard deviation for continuous variable. 
Table 2: Predictors of BMI progression in patients with MDR-TB, multivariate linear mixed random-effect regression.

\begin{tabular}{llccc}
\hline Effects & & Estimate & SE & p-value \\
\hline Intercept & Mean BMI & 15.79883 & 0.54076 & 0.00000 \\
\hline & Treatment success (yes) & 1.73193 & 0.58372 & 0.00301 \\
\hline & Lung Cavities on X-ray (yes) & 1.47195 & 0.76554 & 0.05451 \\
\hline Slope & Time & 0.04917 & 0.09200 & 0.59303 \\
\hline & Interaction Time - Treatment success (yes) & 0.21736 & 0.09380 & 0.02049 \\
\hline & Interaction Time - Lung Cavities on X-ray (yes) & -0.18032 & 0.06664 & 0.00682 \\
\hline
\end{tabular}

Data are given as mean (SE); $\mathrm{SE}=$ standard error; Bolded $\mathrm{p}$ values were $<0.05$, which means the corresponding factor was significantly associated with the BMI progression.

Table 3: Description of the posterior classification from the BMI latent class model according to the baseline characteristics for MDR-TB patients. 


\begin{tabular}{|c|c|c|c|}
\hline Characeristics & $\begin{array}{l}\text { Slow BMI progression } \\
\qquad(n=22) *\end{array}$ & $\begin{array}{l}\text { Rapid BMI progression } \\
\qquad(n=121)^{\star}\end{array}$ & p-value \\
\hline Age at baseline & $37.6(13.8)$ & $32.6(10.7)$ & 0.1002 \\
\hline Sex (female) & $15(68.2)$ & $33(27.3)$ & 0.0004 \\
\hline BMl $\left(\mathrm{kg} / \mathrm{m}^{2}\right)$ & $15.8(2.5)$ & $17.9(2.5)$ & 0.9989 \\
\hline Initial sputum smear (positive) & $19(86.4)$ & $108(89.3)$ & 0.2471 \\
\hline Initial sputum culture (positive) & $15(79.0)$ & $104(89.7)$ & 0.1156 \\
\hline HIV status (positive) & $13(59.1)$ & $22(18.3)$ & 0.0001 \\
\hline History of TB treatment (new case) & $9(40.9)$ & $18(14.9)$ & 0.0061 \\
\hline Treatment adherence (no) & $14(63.6)$ & $4(3.3)$ & $<.0001$ \\
\hline Depression (yes) & $4(18.2)$ & $1(0.83)$ & 0.0002 \\
\hline Chest pain (yes) & $14(63.6)$ & $55(45.5)$ & 0.0550 \\
\hline Cough (yes) & $20(90.9)$ & $120(99.2)$ & 0.0586 \\
\hline Dyspnea (yes) & $15(68.2)$ & $34(28.1)$ & 0.0004 \\
\hline Nausea (yes) & $5(22.7)$ & $7(5.79)$ & 0.0175 \\
\hline Hemoglobin count & $9.8(2.4)$ & $10.8(2.1)$ & 0.3888 \\
\hline Platelets count & $377.7(181.2)$ & $384.2(133.5)$ & 0.0474 \\
\hline Lymphocytes count & $1.7(1.6)$ & $1.9(1.4)$ & 0.3289 \\
\hline Neutrophiles count & $5.1(3.0)$ & $4.9(2.6)$ & 0.3083 \\
\hline Creatinine count & $79.5(17.7)$ & $76.5(20.2)$ & 0.4928 \\
\hline Live SGOT count & $32.7(9.5)$ & $29.2(4.1)$ & $<.0001$ \\
\hline Liver SGPT count & $34.2(6.3)$ & $32.2(4.7)$ & 0.0657 \\
\hline
\end{tabular}

Data are shown as the mean (SD) or number (\%); SD: standard deviation; Bolded p values were $<0.05$, which means the corresponding factor was significantly associated with the BMI subgroups latent class. Groups membership were explained by treatment outcome and lung cavities on X-ray. *Lung Cavities on X-ray at baseline was missing for 22 patients.

Figures 


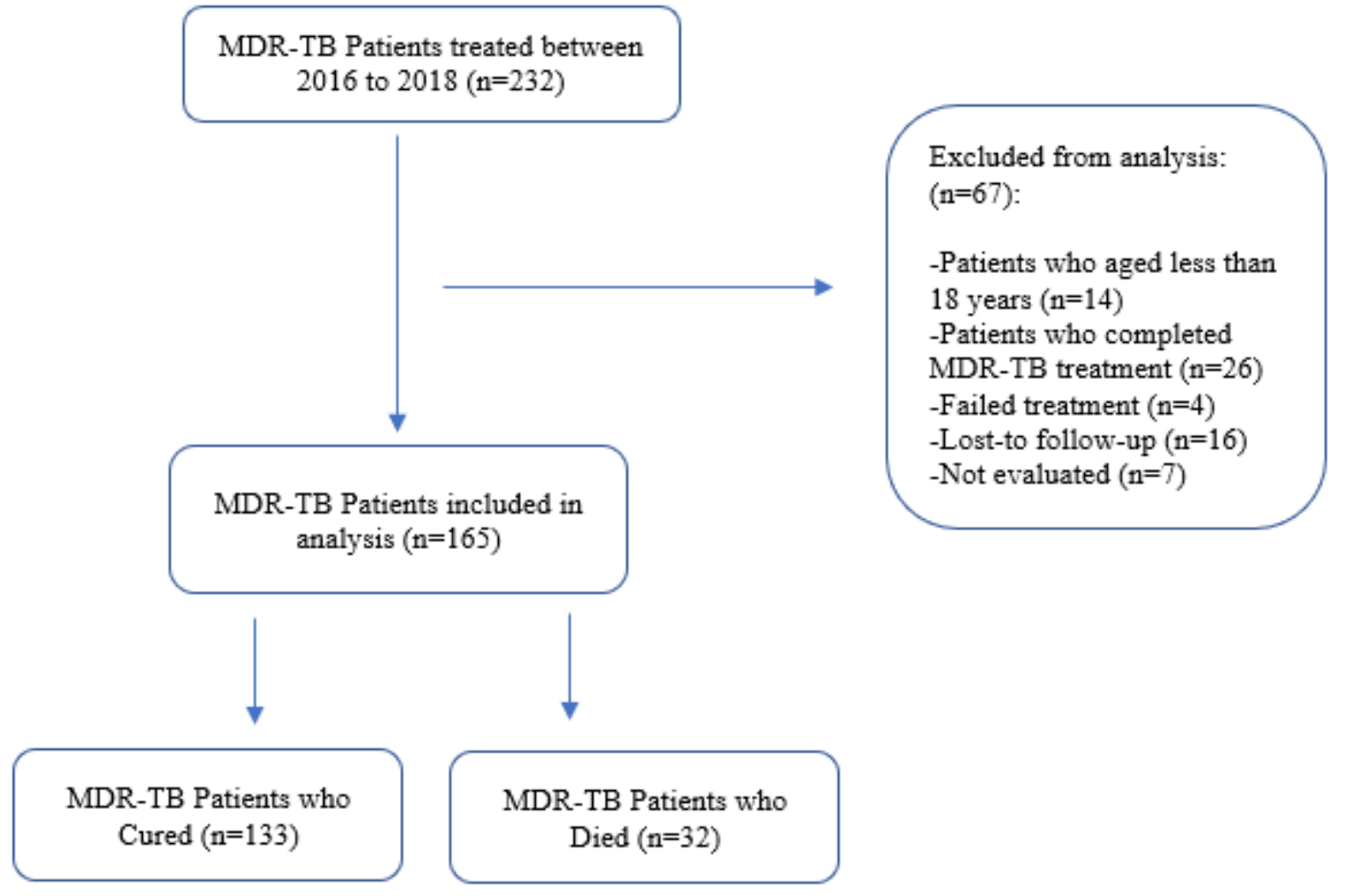

\section{Figure 1}

Flowchart of MDR-TB patients' selection in the study 

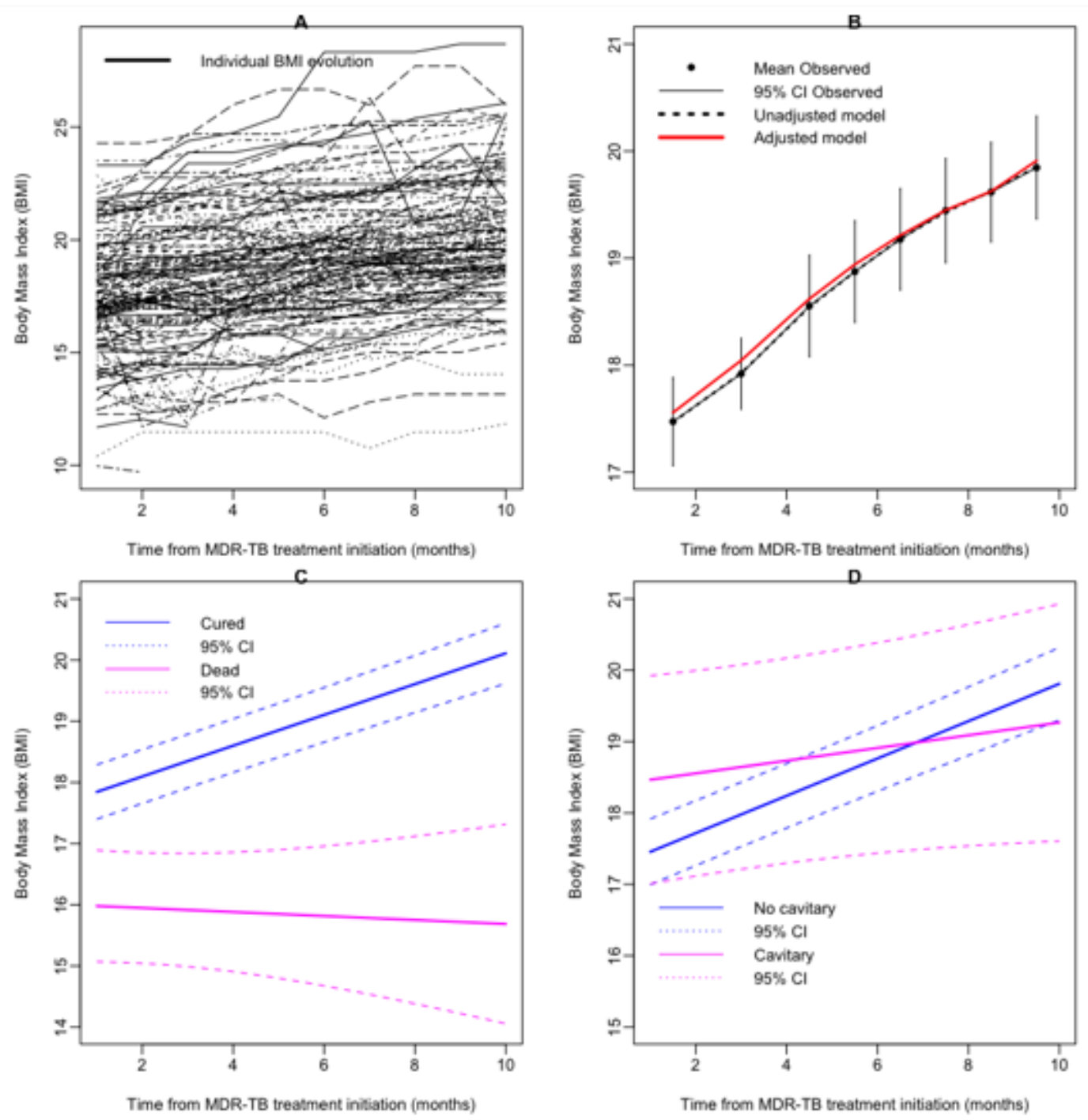

Figure 2

(A) Individual MDR-TB patients' evolution in body mass index (BMI) (black lines), (B) mean increase in BMI (black dots) and 95\% $\mathrm{Cl}$ (black line), unadjusted prediction from linear mixed model (LMM) (dashed line) and adjusted for treatment outcome and lung cavities on X-ray prediction from LMM (red line); (C) Prediction of BMI progression according to the MDR-TB treatment outcome (solid lines): cured (blue line), dead (magenta line), dashed lines were the $95 \% \mathrm{Cl}$ of the prediction from LMM; (D) Prediction of BMI progression according to the lung cavities on X-ray (solid lines): absence of lung cavities (blue line), presence of lung cavities (magenta line), dashed lines were the $95 \% \mathrm{Cl}$ of the prediction from LMM. 


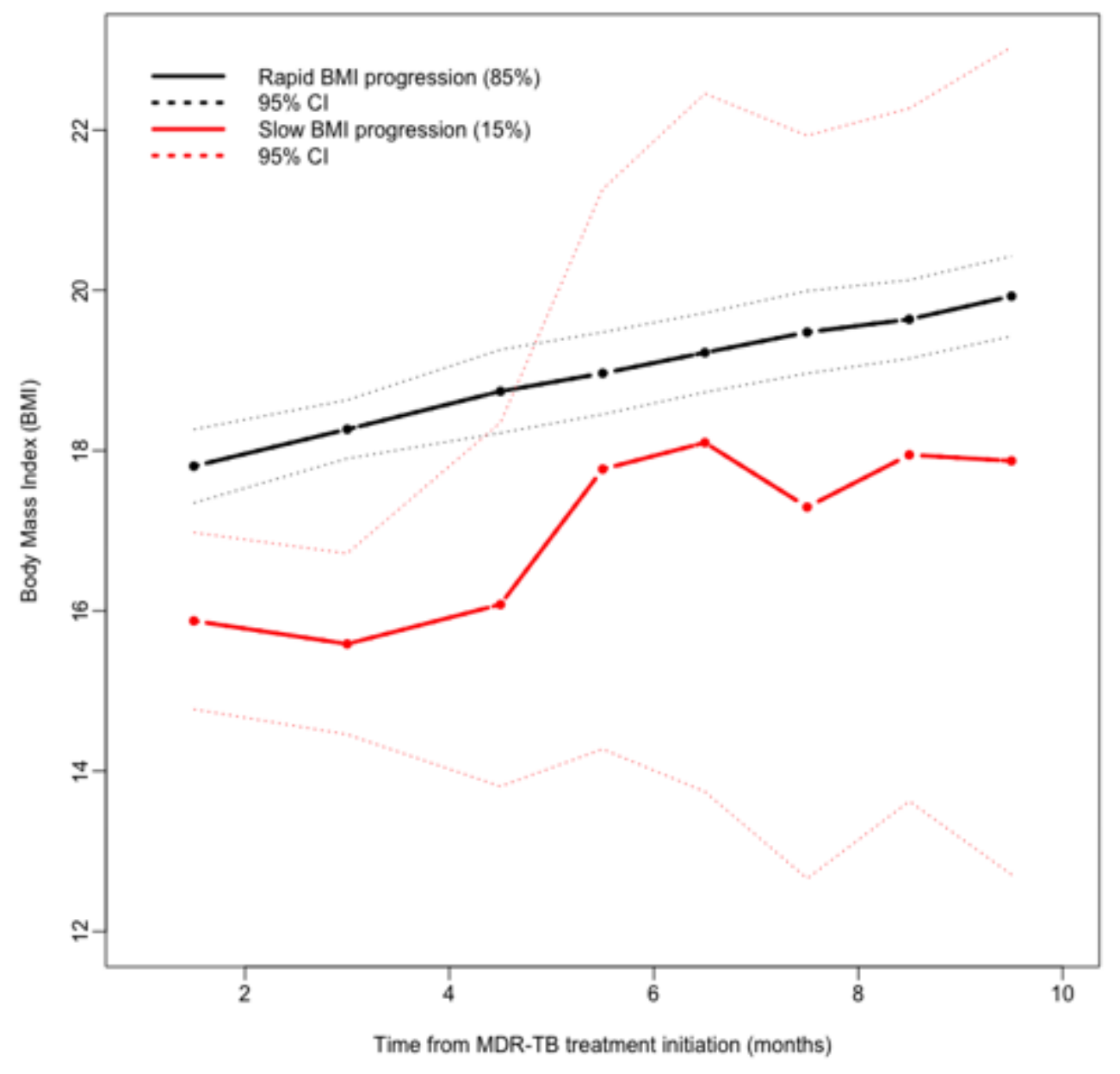

Figure 3

BMI group trajectories in patients with MDR-TB. Weighted patients-specific predictions of BMI trajectories are illustrated according to a latent class mixed model equation: rapid BMI progression (black line) $=17.6$ $+0.26 *$ time; slow BMI progression (red line) $=16.4$ - 0.50*time; dashed lines corresponded to the $95 \%$ confidence interval $(\mathrm{Cl})$ predictions. Groups membership were explained by treatment outcome and lung cavities on X-ray. The width of the IC is due to the fact that there are few patients at the end of the followup in the Slow BMI group. 

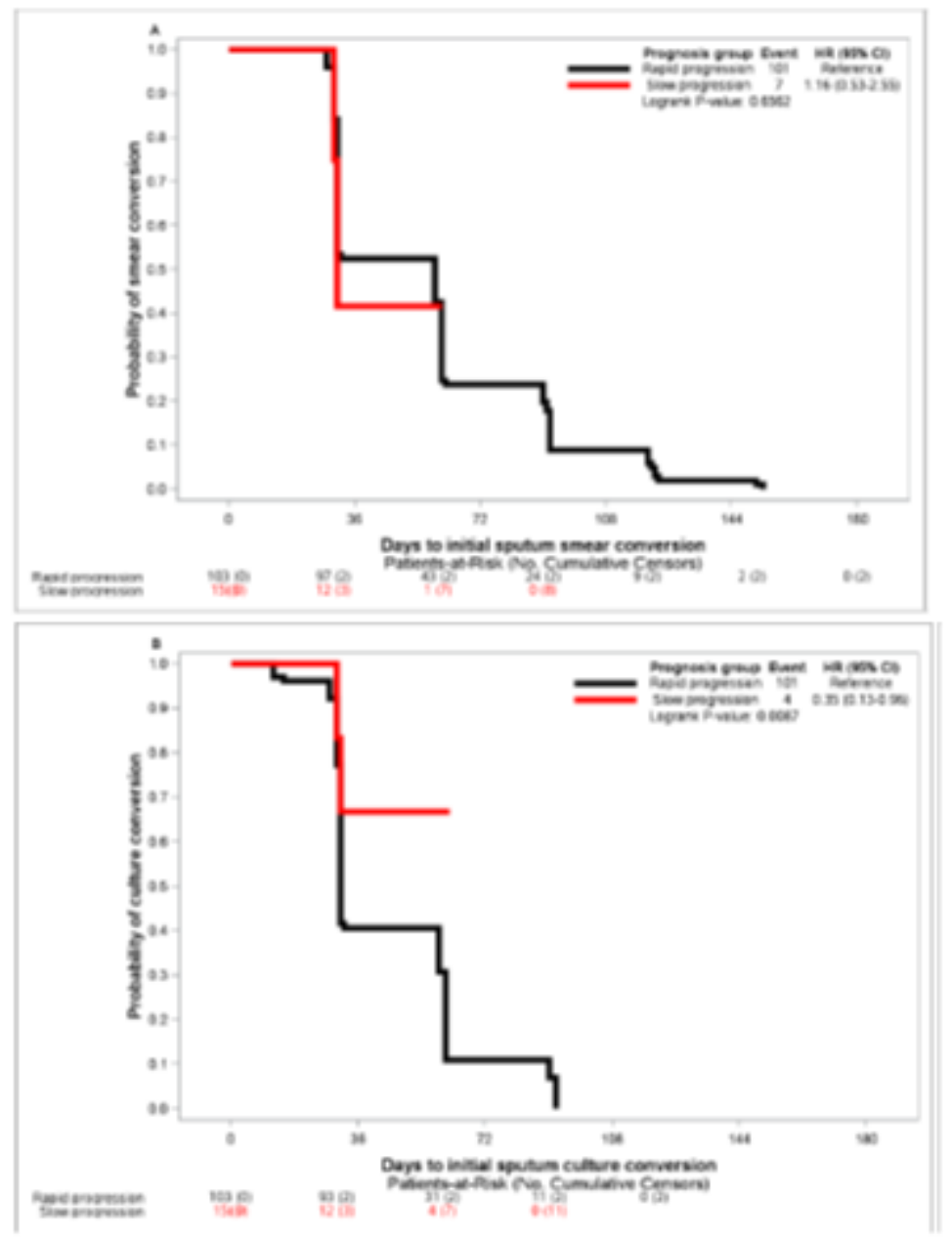

Figure 4

Time-to sputum smear and culture conversions according to the characterization groups from BMI latent classes progression. Only patients who had a positive baseline sputum smear and culture were included in this analysis. 47 patients with negative baseline sputum smear and/or culture were excluded.

\section{Supplementary Files}

This is a list of supplementary files associated with this preprint. Click to download.

- Appendix.docx 